\title{
MODEL PREDICTIVE CONTROL OF A SIMULATED MOVING BED
}

\author{
N. Dejardin ${ }^{*, 1}$, M. Alamir ${ }^{* *}$, J.P. Corriou * \\ * Laboratoire des Sciences du Génie Chimique, \\ LSGC-CNRS-ENSIC, 1 rue Grandville, BP 451, 54001 Nancy \\ Cedex, FRANCE \\ ** Laboratoire d'Automatique de Grenoble, ENSIEG, Domaine \\ Universitaire, BP46, 38402 Saint Martin d'Hères Cedex, \\ FRANCE
}

\begin{abstract}
Chromatographic processes for performing difficult separations such as enantiomers separation with high purity are an expanding technology. An interesting alternative to the expensive conventional batch chromatography is the true moving bed, a counter-current continuous process. However, for feasibility reasons, the TMB cannot be realized. The simulated moving bed (SMB) is realized in practice by connecting several single chromatographic columns in series and simulating the movement of solid by cyclic switching of the inlet and outlet ports. The complex dynamics, important non linearity, and multivariable character of the SMB make its control a challenging task. At this stage of the study, model predictive control (MPC) under the form of quadratic dynamic matrix control (QDMC) is applied on the TMB and the SMB. The numerical simulations of reference trajectory tracking performed show that the QDMC is well adapted for TMB control while the control of the SMB remains improvable. Copyright (c) 2005 IFAC
\end{abstract}

Keywords: predictive control, true moving bed, simulated moving bed, separation, chromatography.

\section{INTRODUCTION}

In several areas of chemical engineering, the fine chemical industry is considered to be the most promising market for the near future. In the pharmaceutical industry (Haag et al. 2001), in the oil industry (Couenne et al. 2001), (Mazzotti et al. 1996), (Kloppenburg and Gilles 1999), or in the food industry (Klatt et al. 2002), pure products are required for several reasons (tests, marketing, or for further processes). Although it is generally possible to develop specific synthesis or separation procedures, the development of such processes is costly and time consuming. Hence, in the concern to accelerate the marketing, the quality and the investment, preparative chromatog-

\footnotetext{
1 Grant from French Ministry of Research
}

raphy has been used to obtain sufficient product quantities.

However, conventional batch chromatography is relatively inefficient in terms of quantities and solvent consumption, as this latter can be very expensive. The separation by means of a continuous counter-current process as the true moving bed process (TMB) could lead to significant benefits. In the TMB, the countercurrent movement of the liquid and the solid phase is performed in order to increase the adsorption and desorption phenomena taking place between these two phases. As the movement of solid particles would result in a deterioration of the solid, the TMB process is unusable in practice. The simulated counter-current moving bed using a fixed bed of adsorbent will not face this degradation problem. The simulated moving 
bed (SMB) is realized by connecting several single chromatographic columns in series and the countercurrent movement of this moving bed is simulated by switching cyclically the set of inlet and outlet valves in the direction of the liquid flow. Owing to its decisive advantages over the batch chromatography, this continuously operated process makes even the separation of a racemic mixture into its enantiomers potentially more attractive than an enantioselective synthesis.

Among many different applications, the simulated moving bed has been used for the separation of hydrocarbons like xylene (Couenne et al. 2001), paraffins (Mazzotti et al. 1996) or aromatics (Kloppenburg and Gilles 1999), in the food industry for glucose and fructose separation (Klatt et al. 2002), also for sucrose separation from molasses, in the pharmaceutical industry for the separation of enantiomers of chiral drugs (Haag et al. 2001). It must be noted that the transfer of the simulated moving bed technology used at large scale for example for xylene separation to the separation of racemic mixtures is not straightforward. Indeed, the conditions and requirements (product quantities and purities, characteristics of the phases) are very different. The main problem for operating successfully the simulated moving bed is the selection of optimal operating conditions, in particular the flow rates in each section. Thus the design of controllers in order to maintain the process operation close to the optimum whatever the present constraints and disturbances is of prime importance. These difficulties require the development of suitable process models and advanced controllers.

In the chemical process industry, model predictive control is a very well accepted technique for controlling multivariable constrained systems (Qin and Badgwell 1996). This is due to the ability of the model predictive control to handle the important features of process control problems such as multivariable dynamics and constraints through an intuitive timedomain formulation. Different techniques have been developed since early versions of IDCOM (Richalet et al. 1978) and Dynamic Matrix Control (Cutler and Ramaker 1979), like quadratic dynamic matrix control (Garcia and Morshedi 1986) and even now nonlinear model predictive control (NMPC) (Allgöwer and Zheng 2000).

This paper presents a short description of the models of the TMB and SMB. Then model predictive control under QDMC form is recalled. Finally simulation results of control of the TMB, then the SMB, are given and commented.

\section{MOVING BEDS}

\subsection{True Moving Bed}

The true moving bed is characterized by a liquid stream and a solid counter-current (Figure 1). It is used as the reference process for the simulated moving bed. The inputs and outputs split the process into four zones: assuming a feed mixture of two components A and B with B preferentially adsorbed by the solid phase, the zone I is a desorption zone of component $\mathrm{B}$, the zone II is a desorption zone for component $\mathrm{A}$, the zone III is an adsorption zone for component $\mathrm{B}$ and finally the zone IV is an adsorption zone for component A.

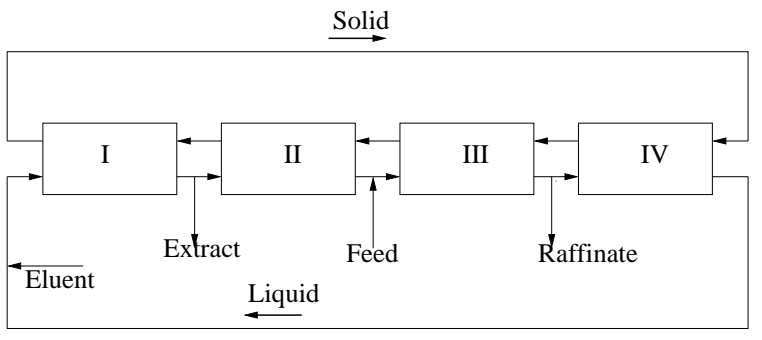

Fig. 1. True moving bed process.

The global mass balance in the zone $j$ for component $i$ is the following

$$
\begin{aligned}
& \epsilon \frac{\partial C_{j}^{i}}{\partial t}+(1-\epsilon) \frac{\partial q_{j}^{i}}{\partial t}+\epsilon v_{j} \frac{\partial C_{j}^{i}}{\partial z} \\
& -\epsilon \mathcal{D} \frac{\partial^{2} C_{j}^{i}}{\partial z^{2}}-(1-\epsilon) v_{s} \frac{\partial q_{j}^{i}}{\partial z}=0
\end{aligned}
$$

$v_{j}$ the fluid velocity in zone $j, v_{s}$ the solid velocity, $\mathcal{D}$ the axial dispersion coefficient, $\epsilon$ the bed porosity, $C_{j}^{i}$ the concentration of component $i$ in the liquid phase, $q_{j}^{i}$ the concentration of adsorbed component $i$ in the solid phase.

The global mass balance is completed by the mass balance in the solid phase

$$
\frac{\partial q_{j}^{i}}{\partial t}-v_{s} \frac{\partial q_{j}^{i}}{\partial z}=k_{i}\left(q_{j}^{i *}-q_{j}^{i}\right)
$$

with $q_{j}^{i *}$ the concentration of component $i$ at equilibrium with the component $i$ in the liquid phase, $k_{i}$ the overall effective mass transfer coefficient.

Considering the equilibrium state, the concentration in the solid phase $q^{i}$ is related to the concentration in the liquid phase $C^{i}$ by a modified competitive Langmuir isotherm

$$
q^{i}=\lambda_{i} C^{i}+\frac{q^{m i} K^{i} C^{i}}{1+\sum_{j=1}^{n_{c}} K^{j} C^{j}}
$$

with $\lambda_{i}$ the linear equilibrium constant, different from zero in the case of enantiomers. $q^{m i}$ is the saturated solid phase concentration of component $i, K^{j}$ the Langmuir equilibrium constant for component $j$, and $n_{c}$ the number of components.

In practice, the solid flow rate of a true moving bed would lead to the attrition of the solid particles and is not feasible. This problem has been solved by the simulated moving bed configuration. 
In a simulated moving bed (SMB) (Figure 2), the adsorption beds are fixed chromatographic columns gathered in a loop and the counter-current solid movement is simulated by periodical switching of the inlet and output ports in direction of the fluid flow. Thus, the position of the inlet and outlet streams depends on the considered instant.

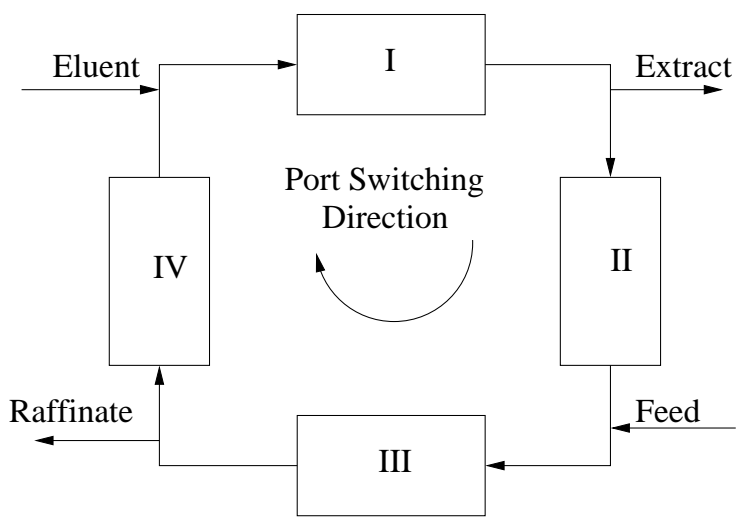

Fig. 2. Simulated moving bed process.

The global mass balance in the zone $j$ for component $i$ is the following

$\epsilon \frac{\partial C_{j}^{i}}{\partial t}+(1-\epsilon) \frac{\partial q_{j}^{i}}{\partial t}+\epsilon v_{j} \frac{\partial C_{j}^{i}}{\partial z}-\epsilon \mathcal{D} \frac{\partial^{2} C_{j}^{i}}{\partial z^{2}}=0(4)$

with $v_{j}$ liquid velocity in the section $j$ (related to the flow rate $F_{j}$ by $F_{j}=\epsilon \Omega v_{j}$ ), $\Omega$ column cross-section.

Because of the null solid velocity, compared to equation (2), the mass balance in the solid phase is modified as

$$
\frac{\partial q_{j}^{i}}{\partial t}=k_{i}\left(q_{j}^{i *}-q_{j}^{i}\right)
$$

The equilibrium equation (3) is unchanged for the SMB.

The mass balance on solid phase (eq. 5) and the Langmuir equilibrium (eq. 3 ) are valid between two commutation instants, i.e. during the period $\left[t_{c}^{k}, t_{c}^{k+1}[\right.$. At time $t_{c}^{k+1}$, a switch occurs so that the liquid flow rate of a zone $j$ during the period $k$ becomes equal to the liquid flow rate of the zone $j-1$ during the period $k-1$.

The liquid flow rates in a section $j$ for the TMB and the SMB are related by noticing that the relative velocity of the liquid with respect to the velocity of the solid in the TMB is equal to the velocity of the liquid in the SMB (Storti et al. 1995). Thus, the internal liquid flow rates $L_{j}$ in the zone $j$ are such that

$$
L_{j}^{S M B}=L_{j}^{T M B}+\frac{\epsilon}{1+\epsilon} Q_{s} \quad(j=1,2,3,4)
$$

with $Q_{s}$ the solid flow rate.
The complex dynamics, the non linearities and the multivariable character make the control of the TMB and particularly the SMB a challenging task. Because of its performances, its flexibility and general industrial acceptance, the model predictive control has been chosen under the quadratic dynamic matrix control form (Garcia and Morshedi 1986). In the model predictive control, the sequence of the manipulated inputs is computed over a control horizon $H_{c}$ so that the predicted outputs follow the reference trajectory over a prediction horizon $H_{p}$. At each prediction step, the first new calculated input is implemented. In QDMC, the process model used for predictions is based on the step responses with respect to a given steady state.

In the case of a single input single output system, the output prediction based on past and future inputs is decomposed as

$$
\begin{aligned}
& \hat{y}(k+l \mid k)=\underbrace{y_{s s}+\sum_{i=l+1}^{M} h_{i} \Delta u(k+l-i)+}_{\text {effect of past inputs }} \\
& \underbrace{\sum_{i=1}^{l} h_{i} \Delta u(k+l-i)}_{\text {effect of future inputs }}+\underbrace{\hat{d}(k+l \mid k)}_{\text {predicted disturbances }}
\end{aligned}
$$

where the $h_{i}$ 's are the step response coefficients, and $M$ is the model horizon, $y_{s s}$ is the open-loop steadystate output.

The output prediction based on past inputs is noted

$$
y^{*}(k+l \mid k)=y_{s s}+\sum_{i=l+1}^{M} h_{i} \Delta u(k+l-i)
$$

The predicted disturbances $\hat{d}(k+l \mid k)$ are taken equal to the actual disturbance $\hat{d}(k \mid k)=y(k)-y^{*}(k \mid k)$.

Over a prediction horizon $H_{p}$, the vector of output predictions is calculated as

$$
\begin{aligned}
& {\left[\begin{array}{c}
\hat{y}(k+1 \mid k) \\
\vdots \\
\hat{y}\left(k+H_{p} \mid k\right)
\end{array}\right]=\left[\begin{array}{c}
y^{*}(k+1 \mid k) \\
\vdots \\
y^{*}\left(k+H_{p} \mid k\right)
\end{array}\right]} \\
& +\mathcal{A}\left[\begin{array}{c}
\Delta u(k) \\
\vdots \\
\Delta u\left(k+H_{c}-1\right)
\end{array}\right]+\left[\begin{array}{c}
\hat{d}(k+1 \mid k) \\
\vdots \\
\hat{d}\left(k+H_{p} \mid k\right)
\end{array}\right]
\end{aligned}
$$

where $\mathcal{A}$ is called the dynamic matrix which incorporates the step response coefficients $h_{i, j}$ corresponding to the various couples $\left(u_{i}, y_{j}\right) . H_{c}$ is the control horizon, in general much smaller than $H_{p}$. It is assumed that the input remains constant after the control horizon $H_{c}$

$$
\Delta u(j)=0 \quad \forall j \geq k+H_{c}
$$


Various types of constraints (hard constraints which must be always respected and soft constraints which can be violated) can be considered: hard constraints affecting the manipulated variables like valve saturations, affecting the controlled variables in order to avoid overshoots, affecting other variables which must be kept within bounds like safety constraints, terminal state constraints (Corriou 2004). Generally, hard constraints are handled as a system of linear inequalities

$$
\boldsymbol{B}_{\boldsymbol{h}} \Delta \boldsymbol{u}(k) \leq \boldsymbol{c}_{\boldsymbol{h}}(k+1)
$$

Finally, while respecting the constraints, the following quadratic criterion is minimized with respect to $\Delta \mathcal{U}(k)$

$$
\begin{aligned}
J= & \|\boldsymbol{\Gamma}[\mathcal{Y}(k+1 \mid k)-\mathcal{R}(k+1 \mid k)]\|^{2}+ \\
& \|\boldsymbol{\Lambda} \boldsymbol{\Delta} \mathcal{U}(k \mid k)\|^{2}
\end{aligned}
$$

submitted to the constraints (11). In this way, it is a quadratic programming problem. $\mathcal{R}$ is the reference trajectory over the prediction horizon, $\boldsymbol{\Gamma}$ and $\boldsymbol{\Lambda}$ are weight matrices.

Furthermore, soft constraints are often considered with respect to the output variables rather than hard constraints. In this case, a penalty function $J_{p e n}$ is added to the criterion (12)

$$
\begin{aligned}
& J_{\text {pen }}=\sum_{i} \mathcal{W}_{i}\left(\frac{\mathcal{F}_{s i}+\left|\mathcal{F}_{s i}\right|}{2}\right)^{2} \\
& \text { with: } \quad \mathcal{F}_{\boldsymbol{s}}=\boldsymbol{B}_{\boldsymbol{s}} \Delta \boldsymbol{u}(k)-\boldsymbol{c}_{\boldsymbol{s}}(k+1)
\end{aligned}
$$

where $\mathcal{W}$ is a weight vector, $\boldsymbol{c}_{\boldsymbol{h}}$ and $\boldsymbol{c}_{\boldsymbol{s}}$ are respectively hard and soft constraints vectors.

The choice of the manipulated inputs should respect physical constraints. As the mass transfer is the key phenomena, the liquid flow rates in the different sections of the SMB appear to be the most natural variables. However, concerning the switching period for the SMB which is a manipulated input, the equivalent solid flow rate of a TMB corresponding to the switching period has been used to represent it. Three other inputs must be chosen. In order to set up convenient economical constraints, the most common used inputs are the solvent flow rate, extract flow rate and the recycle flow rate.

The chosen controlled outputs are the purities at the outlets of the process. However, due to the switches, the instantaneous measured concentrations are not steady even for constant inputs and decrease rapidly during a switching period. Consequently, the measured outputs are obtained by the average purities over one switching period

$$
\bar{y}_{i}(k)=\frac{\int_{t_{k-1}}^{t_{k}} C_{i} Q_{o}}{\sum_{j=1}^{n_{c}} \int_{t_{k-1}}^{t_{k}} C_{j} Q_{o}}
$$

where $Q_{o}$ is the appropriate output flow rate and $n_{c}$ the number of components.

\section{SIMULATION RESULTS}

The operating data for the TMB and the SMB are given in Table A.1. In order to compare how the Quadratic Dynamic Matrix controller performs with the TMB and the SMB, the same set points have been imposed on both processes. A time 0 , from a given steady state regime, the controller is switched on, then at time 20000 s, the set point 2 changes to 0.99 , at time 10000 s the set point 1 change to 0.98 , at time 150000 s the set point 1 changes to 0.99 , at time 250000 , the set point 1 is changed to its original value. Thus, high purities are required. From those changes, the multivariable couplings should appear clearly on the inputs and outputs.

\subsection{True Moving Bed control}

First, Model Predictive Control is applied on the simpler TMB as, in a positive case, the control law can be later transposed to the SMB. The outputs of the TMB are the instantaneous purities.
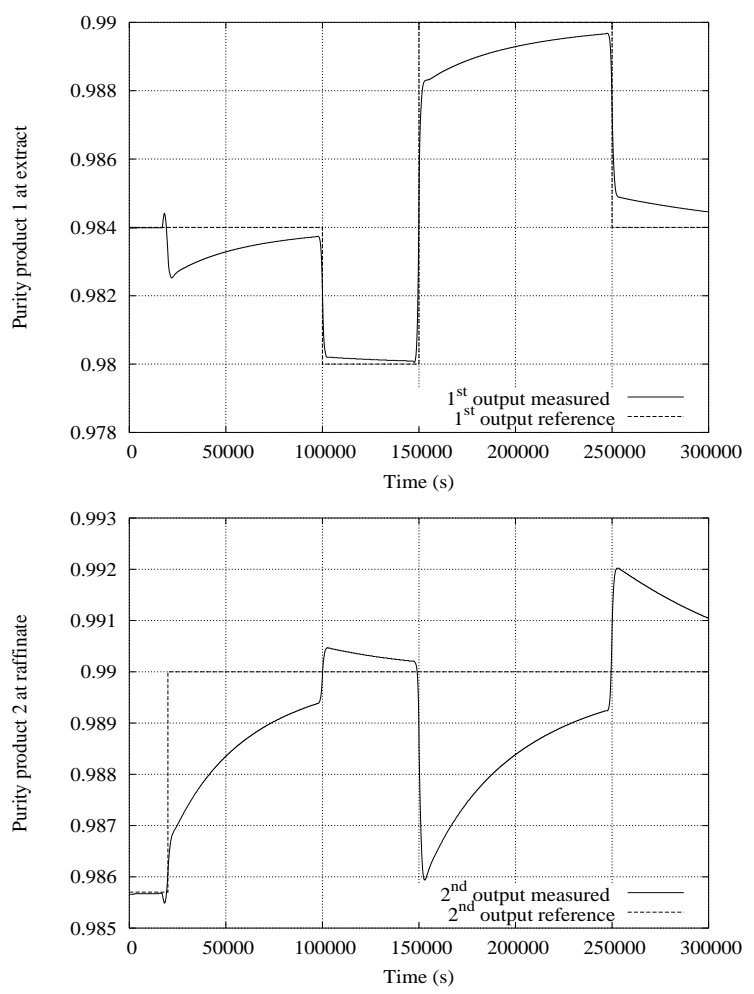

Fig. 3. Purities and set points at the extract (top), and raffinate (bottom) in the case of the true moving bed quadratic dynamic matrix control.

The outputs of the true moving bed process controlled by the quadratic dynamic matrix control (Figure 3 ) follow the desired set points and the inputs are reasonably smooth (Figure 6). The outputs could move faster towards the set points by increasing the weights on the outputs in the criterion at the expense of more moves of the manipulated inputs. 

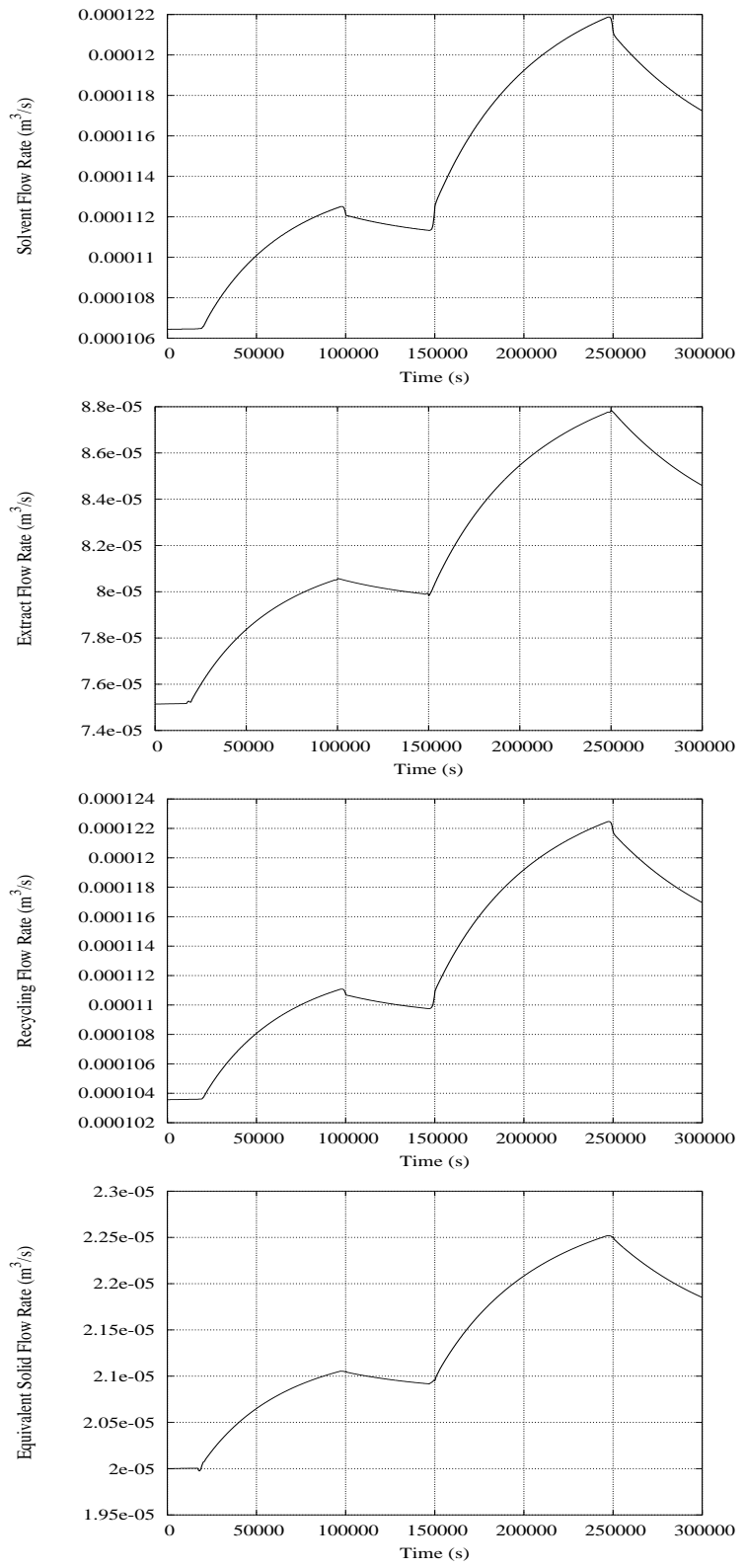

Fig. 4. Solvent, extract, recycle, solid flow rates (from top to bottom) in the case of the true moving bed quadratic dynamic matrix control.

\subsection{Simulated Moving Bed control}

In the SMB control, the outputs are redefined as the mean purities according to equation (14) because of the switching of the valves. They are the controlled outputs shown in Figure 5. Furthermore, in the SMB, there is no solid flow rate, and in reality the switching period is not constant. On Figure 6, an equivalent solid flow rate has been defined for convenience reasons. The outputs are a little fluctuating around the mean purity trajectories, however their behaviour is very acceptable. Also the manipulated inputs could be easily implemented on a real SMB.
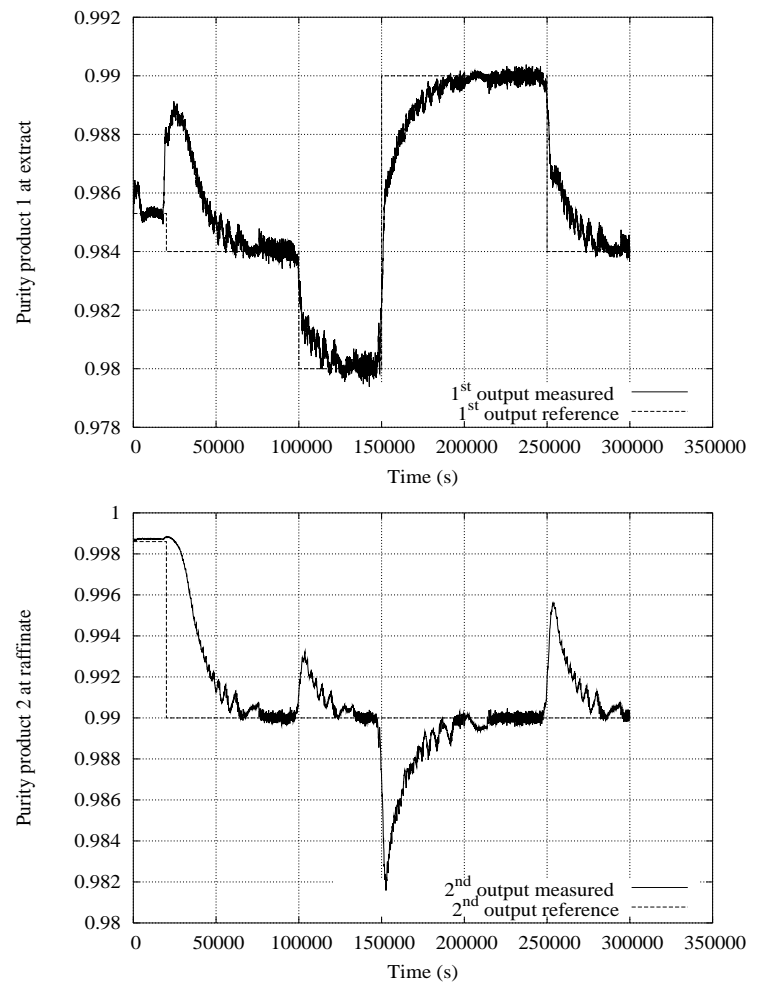

Fig. 5. Purities and set points at the extract (top) and raffinate (bottom) in the case of the simulated moving bed quadratic dynamic matrix control.

\section{CONCLUSION}

The simulated moving bed is a promising technology for both large scale and preparative production in chemical and pharmaceutical industry. In the present study, the Quadratic Dynamic Matrix Control of the simulated moving bed gives a very acceptable behaviour which presents of course large similarities with the controlled TMB. High purities can be achieved and the proposed control strategy could be implemented in real practice.

Because of the very tight operating domain of the SMB and its highly nonlinear character, the design of a SMB (Storti et al. 1993) is delicate. The previous authors have defined design parameters which better represent the SMB separation performance than the usual flow rates. Consequently, defining constraints and especially soft constraints concerning the outputs is not straightforward. As the fundamental difference between violating the constraints and following the references is narrow, the weighting of the different terms of criterion (13) is not obvious. A too weak weight vector $\mathcal{W}$ compared to the $\Gamma$ weight matrix will not efficiently penalize the criterion in order to respect the soft constraints. On the other hand, a too large weight vector $\mathcal{W}$ compared to the $\Gamma$ weight matrix will penalize so much the criterion that the soft constraints are treated as hard constraints. Further work will also deal with the consideration of the physically operating domain into the soft constraints to be incorporated in the penalty function. 

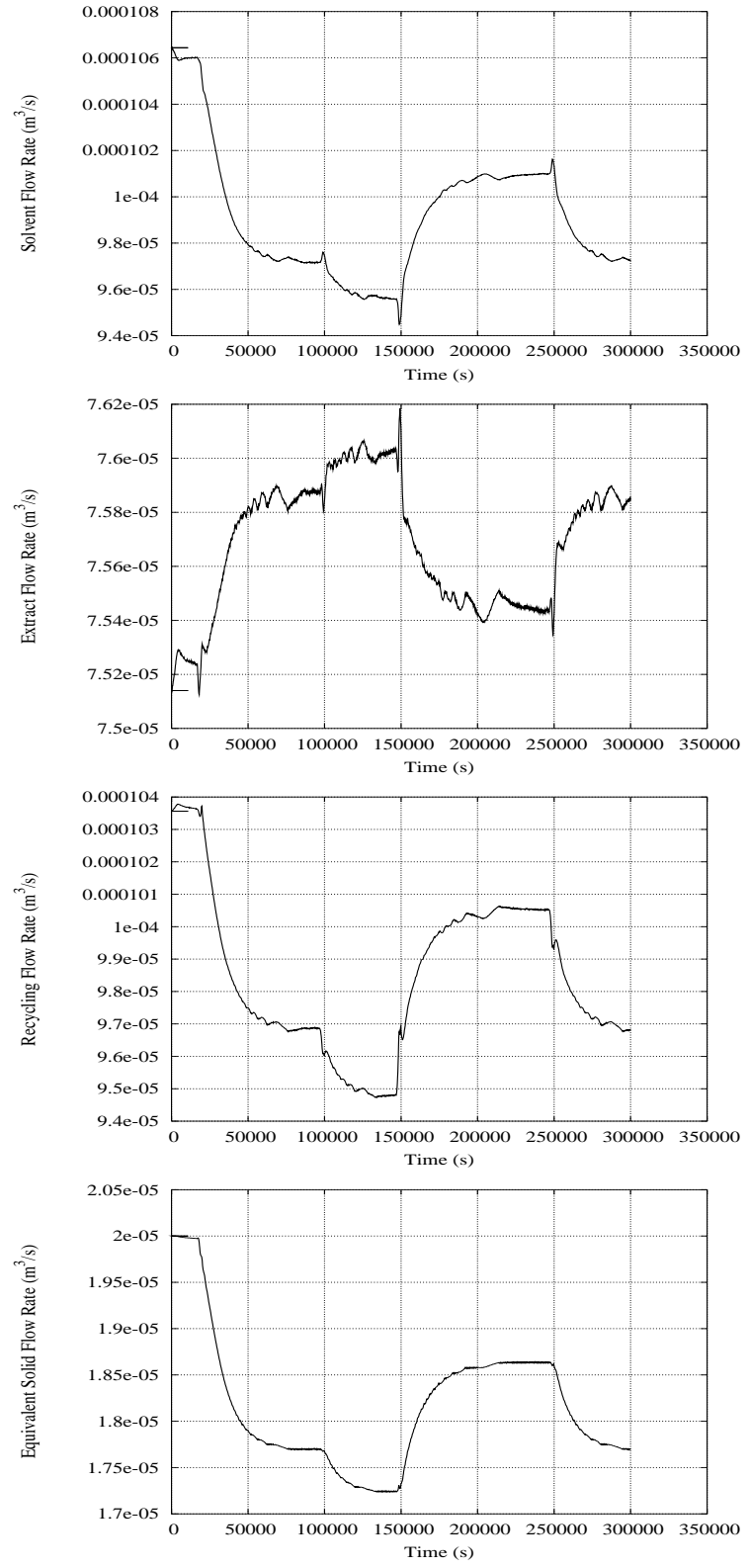

Fig. 6. Solvent, extract, recycle, equivalent solid flow rates (from top to bottom) in the case of the simulated moving bed quadratic dynamic matrix control.

\section{REFERENCES}

Allgöwer, F. and Zheng, A., Eds.) (2000). Nonlinear Model Predictive Control. Birkhäuser. Basel.

Corriou, J. P. (2004). Process Control - Theory and applications. Springer Verlag. London.

Couenne, N., G. Bornard, J. Chebasier and D. Humeau (2001). Contrôle multivariable d'une unité de séparation des xylènes par lit mobile simulé. Symposium IS A - Le contrôle avancé au service de l'industrie.

Cutler, C. R. and B. L. Ramaker (1979). Dynamic matrix control - a computer control algorithm. In: AIChE Annual Meeting. Houston, Texas.

Garcia, C. E. and A. M. Morshedi (1986). Quadratic programming solution of dynamic matrix control (QDMC). Chem. Eng. Comm. 46, 73-87.
Haag, J., A. Vande Wouwer, S. Lehoucq and P. Saucez (2001). Modeling and simulation of a SMB chromatographic process designed for enantioseparation. Cont. Eng. Practice 9, 912-928.

Klatt, K. U., F. Hanisch and G. Dünnebier (2002). Model-based control of a simulated moving bed chromatographic process for the separation of fructose and glucose. J. Proc. Cont. 12, 203-219.

Kloppenburg, E. and E. D. Gilles (1999). Automatic control of the simulated moving bed process for $c_{8}$ aromatics separations using asymptotically exact input/output linearization. J. Proc. Cont. 9, 41-50.

Mazzotti, M., R. Baciocchi, G. Storti and M. Morbidelli (1996). Vapor-phase smb adsorptive separation of liner/nonlinear paraffins. Ind. Eng. Chem. Res. 35, 2312-2321.

Qin, S. J. and T. A. Badgwell (1996). An overview of industrial model control technology. In: Chemical Process Control-CPC V. Tahoe, California. pp. 232-255.

Richalet, J., A. Rault, J. L. Testud and J. Papon (1978). Model predictive heuristic control: Applications to industrial processes. Automatica 14, 413-428.

Storti, G., M. Mazzotti, M. Morbidelli and G. Carra (1993). Robust design of binary countercurrent adsorption separation processes. AIChEJ. 39, 471-490.

Storti, G., R. Baciocchi, M. Mazzotti and M. Morbidelli (1995). Design of optimal operating conditions of simulated moving bed adsorptive separation units. Ind. Eng. Chem. Res. 34, 288-301.

\section{Appendix A. DATA}

Table A.1. Operating data

\begin{tabular}{|c|c|c|}
\hline Number of columns per section & 2 & \\
\hline Number of batch reactors per column & 20 & \\
\hline Length of a column & 0.50 & $(m)$ \\
\hline Diameter of a column & 0.050 & $(m)$ \\
\hline Porosity $\epsilon$ & 0.4 & \\
\hline Feed Flow Rate & $5.6210^{-6}$ & $\left(\mathrm{~m}^{3} / \mathrm{s}\right)$ \\
\hline Solvent Flow Rate & $106.4410^{-6}$ & $\left(\mathrm{~m}^{3} / \mathrm{s}\right)$ \\
\hline Recycle Flow Rate (TMB) & $103.5610^{-6}$ & $\left(\mathrm{~m}^{3} / \mathrm{s}\right)$ \\
\hline Extract Flow Rate & $75.1410^{-6}$ & $\left(\mathrm{~m}^{3} / \mathrm{s}\right)$ \\
\hline Raffinate Flow Rate & $36.9210^{-6}$ & $\left(\mathrm{~m}^{3} / \mathrm{s}\right)$ \\
\hline Solid Flow Rate & $20.0010^{-6}$ & $\left(\mathrm{~m}^{3} / \mathrm{s}\right)$ \\
\hline & 30.00 & \\
\hline Feed concentration in A & 0.5 & $\left(\mathrm{~kg} / \mathrm{m}^{3}\right)$ \\
\hline Feed concentration in B & 0.5 & $\left(\mathrm{~kg} / \mathrm{m}^{3}\right)$ \\
\hline Max A concentration in adsorbent $q^{m 1}$ : & 5 & $\left(\mathrm{~kg} / \mathrm{m}^{3}\right)$ \\
\hline Max B concentration in adsorbent $q^{m 2}$ : & 5 & $\left(\mathrm{~kg} / \mathrm{m}^{3}\right)$ \\
\hline Langmuir constant $K_{1}$ for A & 1.5 & $\left(\mathrm{~m}^{3} / \mathrm{kg}\right)$ \\
\hline Langmuir constant $K_{2}$ for B & 2.0 & $\left(\mathrm{~m}^{3} / \mathrm{kg}\right.$ \\
\hline
\end{tabular}

